\title{
BMJ Open Long-term incidence trends of HPV- related cancers, and cases preventable by HPV vaccination: a registry-based study in Norway
}

Bo T Hansen, Suzanne Campbell, Mari Nygård

To cite: Hansen BT, Campbell S, Nygård M. Long-term incidence trends of HPV-related cancers, and cases preventable by HPV vaccination: a registry-based study in Norway. BMJ Open 2018;8:e019005. doi:10.1136/ bmjopen-2017-019005

- Prepublication history for this paper is available online. To view these files, please visit the journal online (http://dx.doi. org/10.1136/bmjopen-2017019005).

Received 4 August 2017 Revised 30 November 2017 Accepted 11 January 2018

Check for updates

Department of Research, Cancer Registry of Norway, Oslo, Norway

Correspondence to Dr Bo T Hansen; bo.terning.hansen@ kreftregisteret.no

\section{ABSTRACT}

Objectives Examine long-term incidence trends of human papillomavirus (HPV)-related cancer in Norway, and estimate the number of cancer cases preventable by vaccines against HPV $16 / 18$ or HPV 16/18/31/33/45/52/58.

Design Observational registry-based study. We extracted incident cases of HPV-related cancer during 1953-2015 from the Cancer Registry of Norway. Tumour HPV prevalence estimates from large international metaanalyses or from Norway were used to estimate the protective potential of HPV vaccines.

Participants and setting The Norwegian population. Primary outcome measures Incidence trend analyses during 1953-2015 for squamous cell carcinoma (SCC) of the cervix, vulva, vagina, oropharynx, anus and penis, and adenocarcinoma of the cervix. Additionally, the number of cancer cases preventable by HPV vaccination. Results Among women, incidences of SCC of the anus, oropharynx, vulva and cervical adenocarcinoma increased, while vaginal SCC showed no trend. For these cancers combined, the average annual percentage change (AAPC) during $1953-2015$ was 1.2 (95\% Cl 0.7 to 1.6). The incidence of cervical SCC generally decreased during 1976-2004 and remained stable thereafter. Among men, incidences of SCC of the anus, oropharynx and penis increased. The AAPC during 1953-2015 combined for all male HPV-related cancer was 1.9 (95\% $\mathrm{Cl} 1.3$ to 2.5$)$. A vaccine against HPV 16/18 might yearly prevent 402 (95\% Cl 382 to 420$)$ cancers. A vaccine against HPV 16/18/31/33/45/52/58 might yearly prevent 478 (95\% Cl 464 to 490) cancers, of which 206 (95\% Cl 202 to 209) occur in non-cervical organs, and $113(95 \% \mathrm{Cl} 110$ to 115) occur among men.

Conclusions The incidences of HPV-related cancers that are not effectively prevented by screening have generally increased during 1953-2015. HPV vaccination can prevent a substantial number of cancers in Norway, in cervical and non-cervical organs, among women and men.

\section{INTRODUCTION}

\section{Human papillomavirus and cancer}

Human papillomavirus (HPV) is a necessary cause of cervical cancer ${ }^{1}$ and is aetiologically linked to cancer of the vulva, vagina, anus, penis and oropharynx. ${ }^{2}$
Strengths and limitations of this study

A nationally complete record of cancer cases from a large population.

- Analyses of all potentially human papillomavirus (HPV)-related cancer cases at the cervix, vulva, vagina, penis, anus and oropharynx.

- Extensive period for trend analyses.

- The registry data do not reveal the causes of the incidence trends.

- The fraction of cancers attributable to each HPV type is uncertain for most organs.

HPV-related cancers at these sites represent a substantial burden worldwide, and account for approximately $2.2 \%$ and $7.7 \%$ of all cancers in developed and developing countries, respectively. ${ }^{3}$

There are more than 100 types of HPV, ${ }^{4}$ of which 13 high-risk types are classified as carcinogenic to humans. ${ }^{2}$ HPV is very common in the population, ${ }^{5}$ thus most people are likely to be exposed to HPV at some point during their lifetime. Genital HPV is largely sexually transmitted, and persistent infection with oncogenic HPV may lead to cancer. ${ }^{6}$ The proportion of cancers linked to HPV differs between the anatomical sites that are susceptible to oncogenic HPV, which could reflect differences in tissue types, HPV exposure and susceptibility to other risk factors. HPV is considered a necessary cause of cervical cancer. ${ }^{1}$ A very high proportion of anal and vaginal cancers are also associated with HPV, since HPV is detected in approximately $90 \%$ and $80 \%$ of cancers at these sites, respectively. ${ }^{78}$ In penile and vulvar cancer, the proportion positive to HPV is lower, at approximately $30 \% .{ }^{910}$ There is also evidence of an association between high-risk HPV and cancer in organs of the upper aerodigestive tract, particularly in the oropharynx and tonsil. ${ }^{2}$ The HPV prevalence in oropharyngeal 
cancers is characterised by high geographic variation, from $15 \%$ in Central/South America to nearly $80 \%$ in Norway. ${ }^{11}{ }^{12}$ Carcinogenic HPV16 is by far the HPV type most commonly detected in cancers at all sites, while the relative contribution of other high-risk HPV types differ somewhat between sites, ${ }^{1314}$ indicating that the oncogenic potential and tissue tropism is not equal for all high-risk HPVs.

\section{HPV vaccination}

Three prophylactic vaccines against HPV are available, two of which protect against high-risk types 16 and 18, and one that additionally protects against high-risk types $31,33,45,52$ and 58 . The vaccines can prevent cancer caused by these high-risk HPV types through protection against incident infection. Two of the vaccines additionally protect against low-risk types 6 and 11, which cause genital warts. Clinical trials have shown a very high efficacy of these three vaccines against infection and precancer caused by the vaccine-type HPVs, ${ }^{15-17}$ and evidence of high vaccine effectiveness from real-world settings is currently mounting. ${ }^{18}$ Since none of the HPV vaccines are therapeutic, vaccination is most effective if given before exposure to vaccine-type HPVs, that is, before sexual debut.

\section{Trends in HPV-related cancers}

Cervical cancer accounts for most of the HPV-related cancer cases worldwide. ${ }^{3}$ The incidence of squamous cell cervical cancer has declined substantially in countries with extensive cervical screening, ${ }^{19-21}$ but the incidence of cervical adenocarcinoma, against which screening has less effect, is increasing in many countries. ${ }^{22}$ Consistent temporal increases in incidence are also observed for anal and oropharyngeal cancer, ${ }^{23}$ and some recent studies suggest that the incidence of penile ${ }^{24}$ and vulvar ${ }^{25}$ cancer may also be increasing.

\section{Screening and vaccination in Norway}

Widespread use of cervical screening cytology started in Norway during the 1970s, and an organised cervical screening programme has been effective since 1995. The programme recommends screening every 3 years for women aged 25-69, and issues reminders to women who are overdue for screening. In 2009, Norway initiated a school-based HPV vaccination programme in which all girls are offered the vaccine free of charge in the seventh grade (at age 11-13 years). Girls born in 1997 were the first birth cohort targeted by the programme. During 2016-2018, girls born 1991 or later are also offered free catch-up vaccination, and the programme will be extended to include seventh grade boys from 2018.

\section{Study objectives}

The objectives of this study are to assess the incidence trends of HPV-related cancers in Norway, and the preventive potential of the currently available HPV vaccines in this population.

\section{METHODS}

\section{Registry data}

Cancer data were extracted from the Cancer Registry of Norway (CRN). Since 1953, the CRN has registered virtually all cancer cases in the entire Norwegian population. Notification of cancer diagnoses is compulsory by law, and the CRN receives data from clinicians, pathology laboratories and the Cause of Death Registry, which ensures high data completeness and quality. ${ }^{26}$ In the present study, we focus on cancers occurring at anatomical sites for which there is strong evidence of an association between cancer and high-risk HPV. ${ }^{2}$ Thus, for the period 1953-2015, we extracted data on all incident primary cases of malignant cancer (except basal cell carcinomas) that occurred at the following anatomical sites (International Classification of Diseases, 10th revision topography code): base of tongue (C01), tonsils (C09), oropharynx (C10), anus (C21), vulva (C51), vagina (C52), cervix (C53) or penis (C60), which gave a total of 33997 cases. For each case, the registry data extracted were: tumour topography and morphology, date of diagnosis, patient sex and age at diagnosis. We excluded all cases that were not squamous cell carcinomas (SCC), except in the cervix, where we excluded all cases that were not SCC or adenocarcinomas. A total of 3087 cases were thus excluded. We also excluded 428 cases with unknown morphology. Hence, a total of 30482 cases were included in the analyses. Base of tongue, tonsils and oropharynx were grouped together in the analyses, and are henceforth referred to as 'oropharynx'. Age-specific population size by sex and calendar year was obtained from the National Registry. Data access was granted to address specific research aims, and the project data can be accessed on application to the CRN.

\section{Incidence trends}

We present incidence rates per 100000 person-years, age-standardised by the World Standard Population. ${ }^{27}$ Temporal incidence trends on age-standardised data for each organ/tissue type and for each sex were assessed by joinpoint regression. The analysis identifies periods with distinct linear slopes that can be connected by joinpoints denoting trend changes, and determines how many (if any) joinpoints should be used to best describe trends in the data. ${ }^{28}$ The minimum and maximum number of joinpoints allowed in the time series were zero and four, respectively, and there had to be at least four data points between any joinpoints, and at least three data points from a joinpoint to either end of the data series. The annual percentage change (APC) with $95 \%$ CI was estimated for each trend identified by fitting a linear regression to the logarithm of the rates by calendar year. Under a log-linear model, the rates change at a constant per cent per year, which facilitates comparisons across cancer sites with different incidence rates. We also estimated the average annual percentage change (AAPC) over the whole 63-year period 19532015. For overall analyses with identified joinpoints, the 
AAPC is the average of the individual APCs weighted by the length of each segment. ${ }^{29}$ For analyses with no identified joinpoints during 1953-2015, the AAPC and the APC are the same. We use yearly rates in the trend analyses, with the exception of anal SCC, which is analysed by 3-year moving average rates because some years had no registered anal SCC cases. Significance tests of joinpoint regressions were performed by Monte Carlo permutation with 4499 replicates. Two-sided $\mathrm{P}$ values were considered significant when they were $<0.05$.

\section{HPV attribution}

The CRN does not have data on the HPV status of each tumour. To estimate the number of cases attributable to HPV and to vaccine-type HPV, we multiply the site-specific annual average number of cases for the last 5-year period with corresponding estimates of tumour HPV prevalence from the scientific literature. Because HPV is generally accepted as a necessary cause of cervical cancer, ${ }^{1}$ all cervical cancers were attributed to HPV. For SCC of the vulva, ${ }^{14}$ vagina, ${ }^{8}$ anus ${ }^{7}$ and penis, ${ }^{9}$ and for cervical cancer, ${ }^{30}$ we derived HPV-attributable fractions from the largest meta-analyses that provided worldwide estimates of the type-specific and histology-specific HPV DNA prevalence of HPV 16/18/31/33/45/52/58, and that adjusted for multiple infections of the same sample. Since HPV DNA prevalence for cancer at the oropharynx shows substantial geographical and temporal variation, we used prevalence data from a recent Norwegian study on SCC at this anatomical site. ${ }^{12}$ For the type-specific HPV attribution estimates, multiple infections of the same sample were added to single infections using weighting proportional to the prevalence for each individual type in single infections. ${ }^{30}$ Exact binomial CIs were obtained for the HPV-attributable fractions and for the expected number of cases averted by HPV vaccination.

\section{RESULTS \\ Incidence trends}

The analysis of the cervical SCC incidence trend revealed five distinct periods during 1953-2015, with joinpoints in 1976, 1986, 1991 and 2004 (table 1, figure 1A). An initial increase until the mid-1970s was followed by a general decrease until 2004, and stable rates thereafter. The AAPC $(95 \% \mathrm{CI})$ for the whole period 1953-2015 showed a decrease in incidence of cervical SCC, at -0.7 $(-1.4$ to -0.1$)$. Cervical adenocarcinoma and vulvar SCC, in contrast, had no joinpoints defined in the analyses, and showed consistently increasing incidences over the whole period 1953-2015, with AAPCs of 1.4 (1.1 to 1.7 ) and 0.5 (0.3 to 0.8 ), respectively (table 1 , figure $1 \mathrm{~B}, \mathrm{C}$ ). Vaginal SCC was the rarest HPV-related cancer, and no significant trend was observed, neither for the three periods defined by the analyses nor for the AAPC during 1953-2015 (-0.1 (-2.3 to 2.1); table 1, figure 1D). Anal SCC among women showed the strongest increase of all the HPV-related cancers addressed, with an AAPC of 4.2 (2.7 to 5.8) for the whole 63-year period. Changes in the trend occurred in 1968 and 1978, and the incidence increase was particularly pronounced during the period between these two joinpoints (table 1, figure 1E). Oropharyngeal SCC among women showed a decreasing incidence until 1974, followed by an increase during the subsequent 41 years. For the whole period 1953-2015, a significantly increasing incidence of oropharyngeal SCC among women was observed (AAPC: 1.3 (0.1 to 2.5), table 1 , figure $1 \mathrm{~F}$ ).

Penile SCC showed a consistently increasing incidence during 1953-2015 (AAPC (95\% CI) 0.6 (0.3 to 0.9), table 1 , figure 2A). Anal SCC among men also showed a significantly increasing incidence during 1953-2015 (AAPC: 3.1 $(0.2$ to 6.2$))$. Changes in the incidence trend occurred in 1970, 1975 and 2011, and during the final period (20112015), the incidence of anal SCC among men declined non-significantly (table 1, figure 2B). Oropharyngeal SCC among men showed a similar pattern to that among women, with an overall significantly increasing incidence for the whole period 1953-2015 (AAPC: 2.5 (1.8 to 3.3)), and a joinpoint defined in 1976, when the increase in incidence began (table 1, figure 2C).

The combined trend for HPV-related cancers other than cervical SCC (ie, cervical adenocarcinoma, and SCC of the vulva, vagina, penis, oropharynx (both sexes) and anus (both sexes), none of which are effectively prevented by screening) was increasing during the 63-year period analysed, both for women (AAPC $(95 \% \mathrm{CI})$ for SCC of the vulva, vagina, anus, oropharynx and cervical adenocarcinoma combined: 1.2 (0.7 to 1.6), table 1 , figure $3 \mathrm{~A}$ ), and for men (AAPC for SCC of the anus, oropharynx and penis combined: 1.9 (1.3 to 2.5 ), table 1 , figure $3 \mathrm{~B}$ ). In both cases, the trend did not change significantly during the first decades (1953-1968 for women, 1953-1972 for men), but an extensive period of consistently increasing incidence followed thereafter (1968-2015 for women, 1972-2015 for men).

The total number of incident cases of HPV-related cancer other than cervical SCC increased consistently and more than fourfold during the period analysed, reaching approximately 450 cases diagnosed in 2015 . In contrast, the number of incident cases of cervical SCC was at a similar level in 1953 and in 2015, at approximately 250 cases per year (figure 4). During the first decades analysed, incident cervical SCC vastly outnumbered the other HPV-related cancers combined, but this relationship is now reversed. The shift occurred around the turn of the century (figure 4).

\section{Current HPV vaccine potential}

During the last 5-year period (2011-2015), an average of 644 cases of SCC of the anus, oropharynx, cervix, vulva, vagina, penis and cervical adenocarcinoma occurred annually in Norway. This amounts to $2.0 \%$ of all cancers occurring each year in Norway $(1.0 \%$ and $3.1 \%$ of cancers occurring among men and women, respectively). ${ }^{31} \mathrm{~A}$ 
Table 1 Incidence trend analysest for all HPV-related cancers in Norway 1953-2015

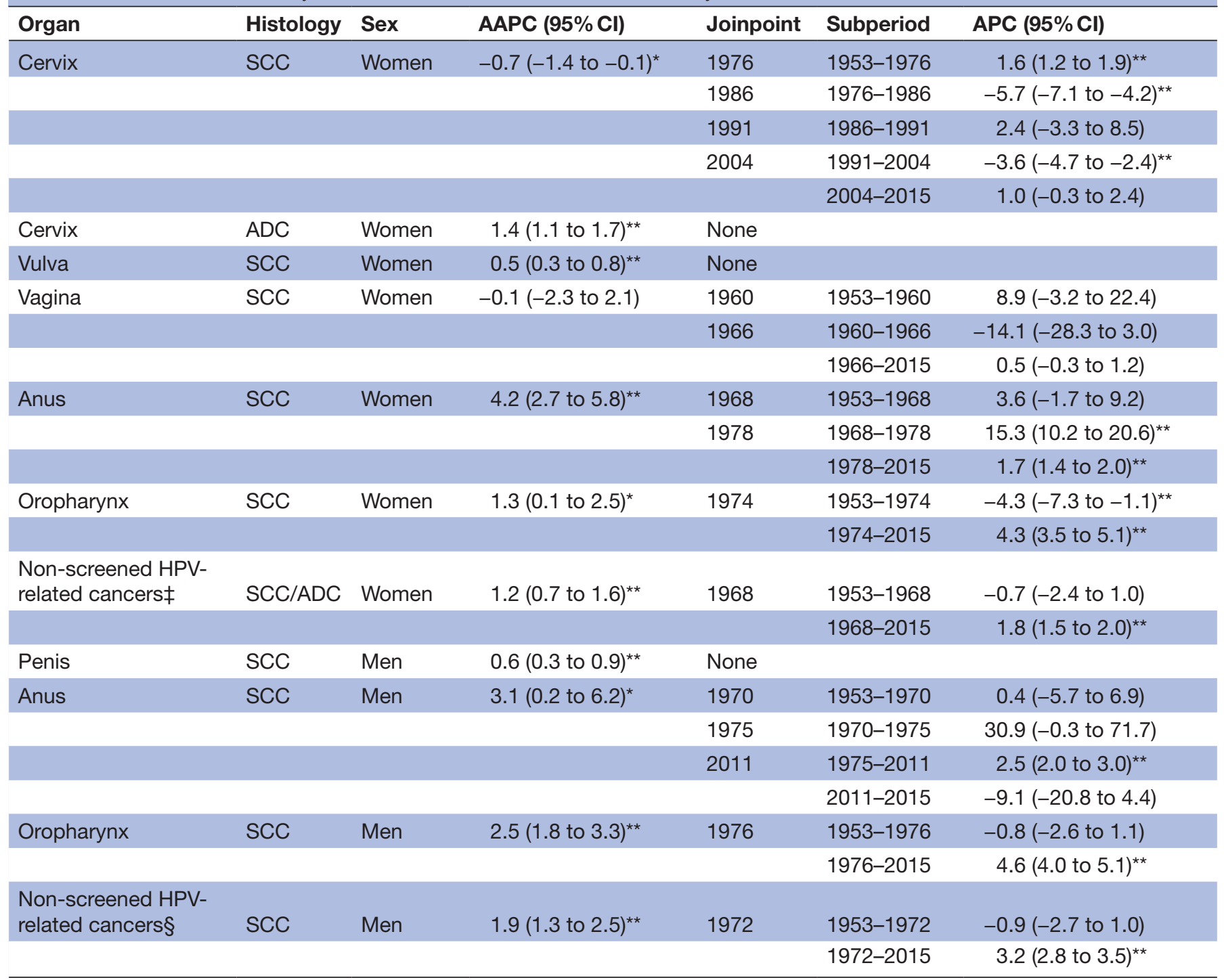

${ }^{*} \mathrm{P}<0.05$.

${ }^{*} \mathrm{P}<0.01$.

†Joinpoint regression analyses.

‡Cervical adenocarcinoma, vulvar SCC, vaginal SCC, anal SCC and oropharyngeal SCC combined.

§Penile SCC, anal SCC and oropharyngeal SCC combined.

AAPC, average annual percentage change for the whole period $1953-2015$; ADC, adenocarcinoma; APC, annual percentage change for subperiod; HPV, human papillomavirus; SCC, squamous cell carcinoma.

total of 521 (95\% CI 500 to 540) of these cases can be attributed to any HPV (table 2).

A vaccine that protects against HPV $16 / 18$ has the potential to prevent 402 (382 to 420) cancer cases per year in Norway, of which 219 (212 to 226) occur in the cervix and the remaining 183 (178 to 188) cases occur in non-cervical organs (table 2). A total of 163 (157 to 169) cases of cervical SCC and 239 (233 to 246) cases of cancer that are not cervical SCC might be prevented each year by a vaccine against HPV 16/18. Moreover, 100 (97 to 104) annual cases of HPV-related cancer among men might be prevented by an HPV 16/18 vaccine (table 2).

A vaccine that protects against HPV $16 / 18 / 31 / 33 / 45 / 52 / 58$ might prevent a total of 478 (464 to 490) cancer cases per year in Norway (table 2).
Cervical cancer accounts for 272 (267 to 276) of these cases, and the remaining 206 (202 to 209) cases occur in non-cervical organs. The most protective vaccine might prevent 207 (203 to 211) annual cases of cervical SCC, and an additional total of 271 (265 to 274) cases of HPV-related cancers that are not cervical SCC. Each year, 113 (110 to 115) cases of cancer among men might be prevented by a vaccine against HPV 16/18/31/33/45/52/58, most of which occur at the oropharynx (table 2 ).

\section{DISCUSSION}

This is the first study estimating the number of cancer cases preventable by vaccines that protect against HPV $16 / 18$ or HPV 16/18/31/33/45/52/58 in Norway. 

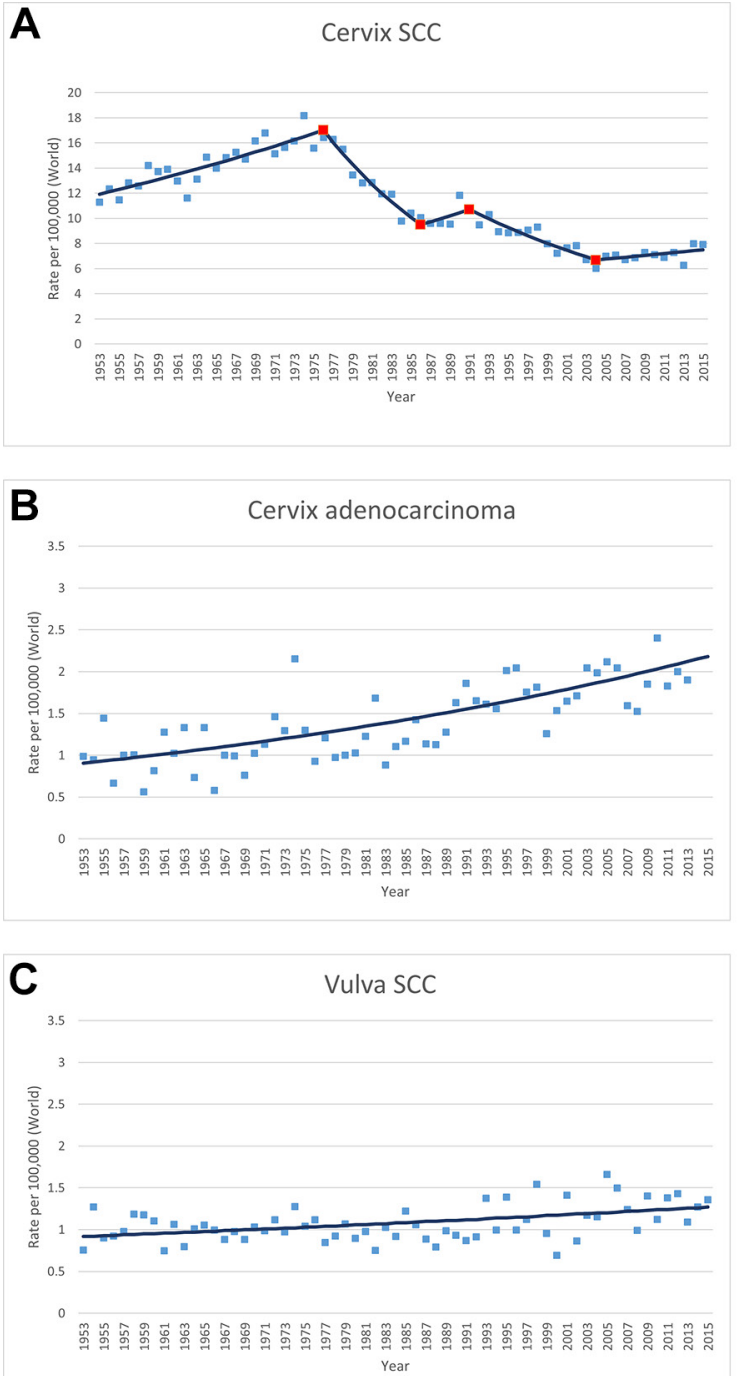
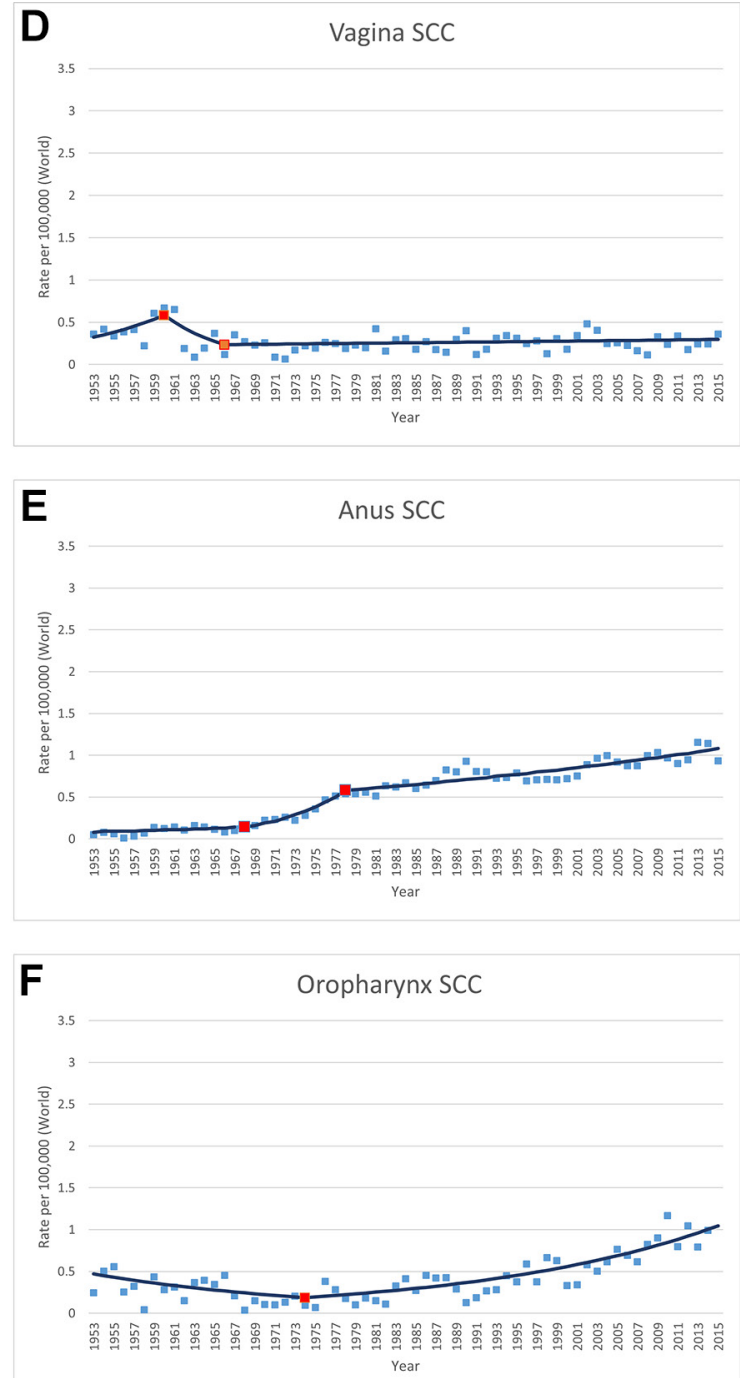

Figure 1 Incidence trends for each human papillomavirus-related cancer-type among women in Norway 1953-2015. Blue points denote observed age-standardised (world) incidence per 100000 women-years. Lines denote the best-fitting trend line, and red points the joinpoints, from joinpoint regression analyses. SCC, squamous cell carcinoma.

It is also among the first studies to present complete nationwide data on long-term incidence trends for cancers at all organs for which there is strong evidence of an association with high-risk HPV. We found that the incidence rates of HPV-related cancers that are not effectively prevented by screening in general have increased in the course of the last 63 years. The only exception to this pattern was for vaginal SCC, where no significant change in incidence was detected. The incidence of cervical SCC has been strongly reduced by screening since the 1970s, and has been stable since 2004. Based on the current incidence of HPV-related cancers in Norway, the total number of incident cases that annually might be prevented by HPV vaccination is substantial. The majority of cancer cases preventable by HPV vaccination are not effectively prevented by current screening.

The incidence trends observed in the present study are generally in line with reports from other countries. Consistently increasing incidence trends for anal cancer, oropharyngeal cancer and cervical adenocarcinoma have been observed in several populations. ${ }^{22}{ }^{32} 33$ In some countries, there is also emerging evidence for an increasing incidence of vulvar and penile cancer. ${ }^{25} 34$ Temporal changes in clinical detection, diagnosis, reporting or coding practice could influence cancer trends, and such artefacts cannot be ruled out as explanations to the trends observed here and in other studies. It is noteworthy, however, that similar trends are observed across populations. Moreover, here, we observe similar trends for cancers across several organs and tissue types, and we generally do not observe abrupt and short-term changes in trends for cancers that are not affected by screening, which would be typical if clinical/registry changes were highly influential.

The risk factor common to these cancers is oncogenic HPV. The generally increasing trend observed in the present study could be caused by increased transmission of HPV at the population level due to changes in sexual behaviour. Population-based studies show that the age at first intercourse has dropped substantially 

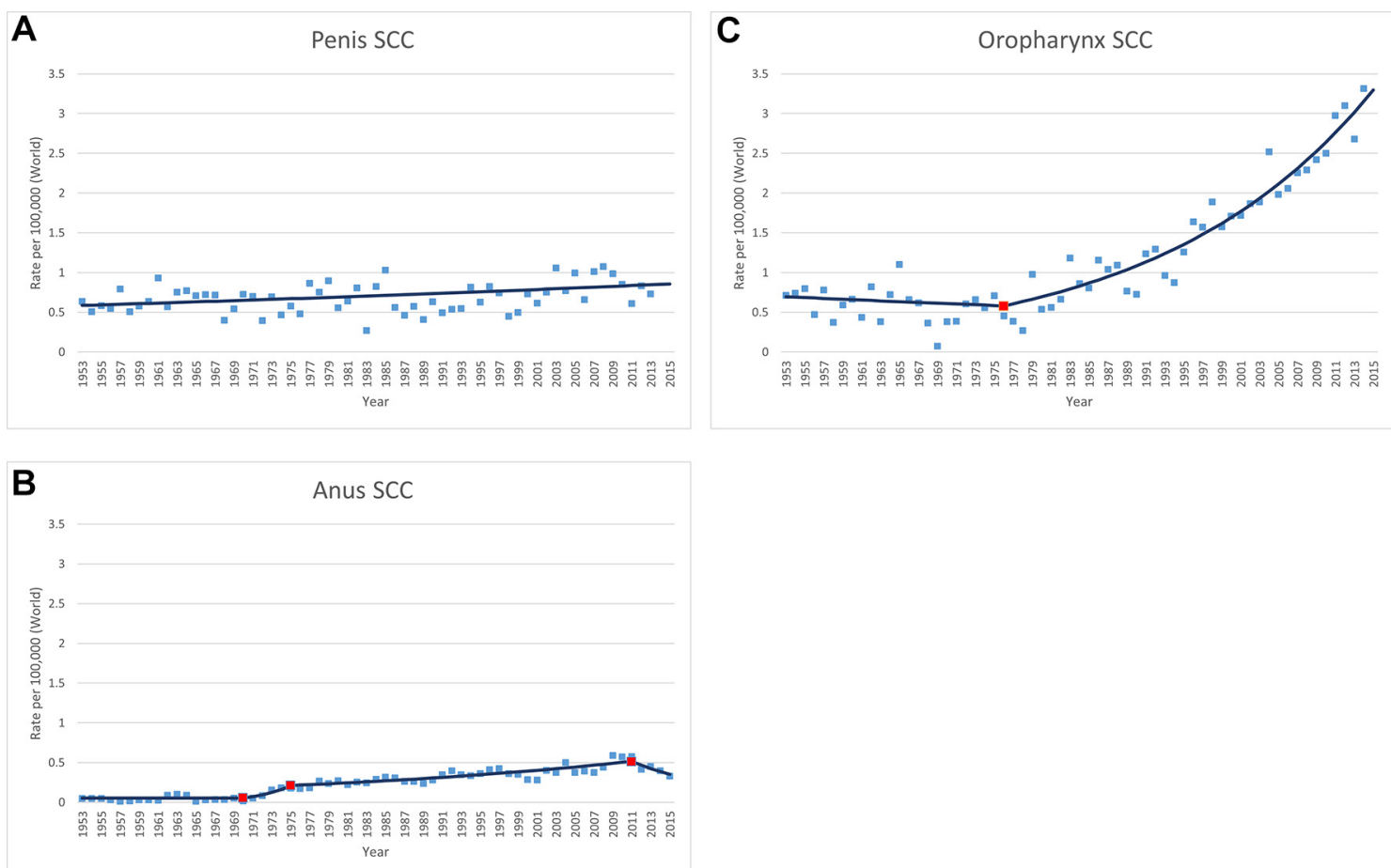

Figure 2 Incidence trends for each human papillomavirus-related cancer type among men in Norway 1953-2015. Blue points denote observed age-standardised (world) incidence per 100000 man-years. Lines denote the best-fitting trend line, and red points the joinpoints, from joinpoint regression analyses. SCC, squamous cell carcinoma.

in Norway during the past century. Compared with the cohort born 1927-1934, the median age at first intercourse was more than 2 years lower among women born during the 1960s and 1970s, while a corresponding 1 year drop was observed among men. ${ }^{35}$ Similar population-based findings of changes in sexual behaviour have also been reported from other countries. ${ }^{36-38}$ We have recently shown that the age at first intercourse is still decreasing among Norwegian women, and that sexual debut at age 14 or younger is becoming more common. Moreover, the number of sexual partners reported per woman in Norway increases with calendar time. ${ }^{39}$ Early sexual debut and more sexual partners are likely to increase transmission of HPV in the population, and are well-established behavioural risk factors for HPV-related cancer. ${ }^{40-42}$ For several of the HPV-related cancers, and for the non-screened cancers combined, a joinpoint was detected during the late 1960s/early 1970s, followed by a long-term increase in incidence. Thus, since HPV-related cancers may take several decades to develop, ${ }^{43}$ a gradual shift in the underlying risk for HPV-related cancers may have started during the 1950s.

Widespread use of the Pap test among Norwegian women started in the early 1970s, and the generally decreasing trend of cervical SCC from 1975 to 2004 was thus most probably caused by cervical cancer screening. ${ }^{20}$ The stable incidence thereafter suggests exhaustion of the potential for further incidence reduction caused by
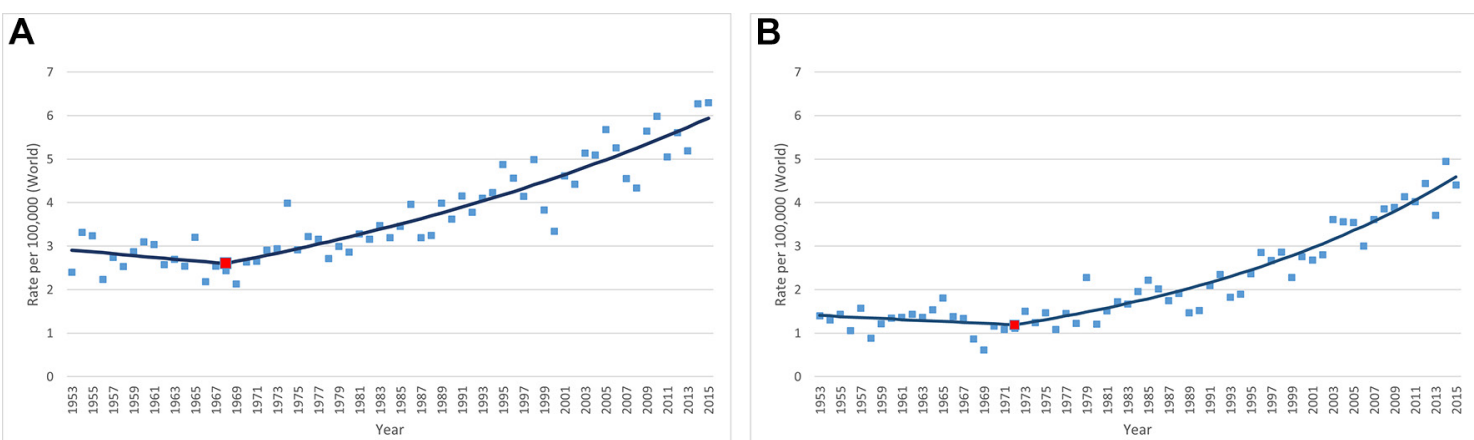

Figure 3 Incidence trends for all non-screened human papillomavirus-related cancer types combined in Norway 1953-2015. (A) Women (combined cancers: cervical adenocarcinoma, anal squamous cell carcinoma (SCC), vaginal SCC, vulvar SCC, oropharyngeal SCC). (B) Men (combined cancers: penile SCC, anal SCC, oropharyngeal SCC). Blue points denote observed age-standardised (world) incidence per 100000 person-years. Lines denote the best-fitting trend line, and red points the joinpoints, from joinpoint regression analyses. 


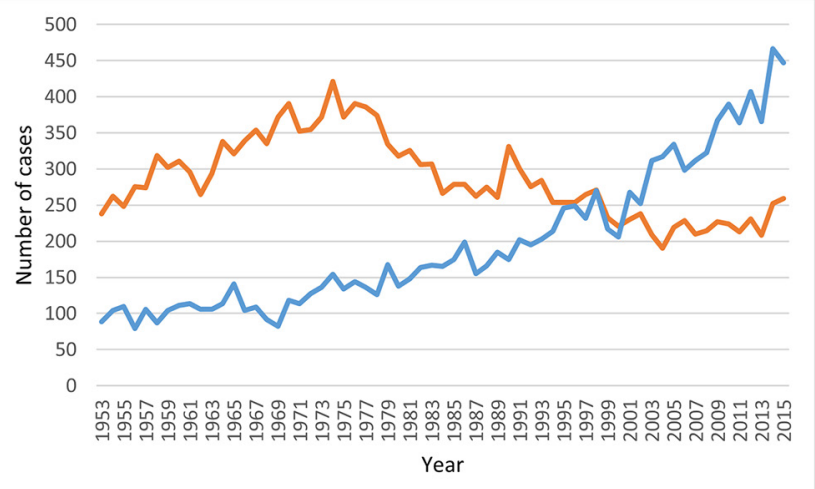

Figure 4 Number of incident cases of cervical squamous cell carcinoma (SCC) (orange) and all other human papillomavirus (HPV)-related cancer combined (blue) in Norway 1953-2015. Combined cancers: cervical adenocarcinoma, vaginal SCC, vulvar SCC, penile SCC, oropharyngeal SCC (both sexes), anal SCC (both sexes).

this intervention in its current form. Of note, the national incidence trend for cervical SCC during 2004-2015 was in fact increasing slightly, and this positive trend just fell short of statistical significance. The stable incidence of vaginal SCC throughout the period analysed could be related to the rarity of this disease, which makes potential trends harder to detect due to the impact of random fluctuations. A potential incidence increase in vaginal SCC, vulvar SCC and cervical adenocarcinoma may also have been curbed to some extent by cervical screening, since all these cancers are preceded by treatable preinvasive lesions that may be detected during gynaecological examination. Moreover, the incidence of oropharyngeal cancer is likely to be higher if estimated among those who have tonsils, since tonsillectomy has been commonly performed for other indications, such as chronic infection, paratonsillar abscess or sleep apnoea.

The remarkable increase in the incidence of HPV-related cancers that are not effectively prevented by screening, and the apparent exhaustion of the cancer-reducing potential of current cervical screening, highlight the importance that HPV vaccination may have for improving public health in the future. If the incidences of HPV-related cancers continue to increase, the future cancer prevention by HPV vaccination will be higher than estimated in this study. In spite of effective screening against cervical SCC, this cancer still has the highest incidence rate among the HPV-related cancer types in Norway, and the highest potential for reduction by HPV vaccination. Moreover, the total number of cervical cancer cases (SCC and adenocarcinoma) accounts for the majority of HPV-related cancer cases in Norway. Nevertheless, the combined number of HPV-related cancer cases occurring at non-cervical sites is also substantial, and HPV vaccination has the potential to prevent a large number of cancer cases in these organs, both among women and among men. Using a vaccine that protects against HPV 16/18/31/33/45/52/58 may prevent a substantially

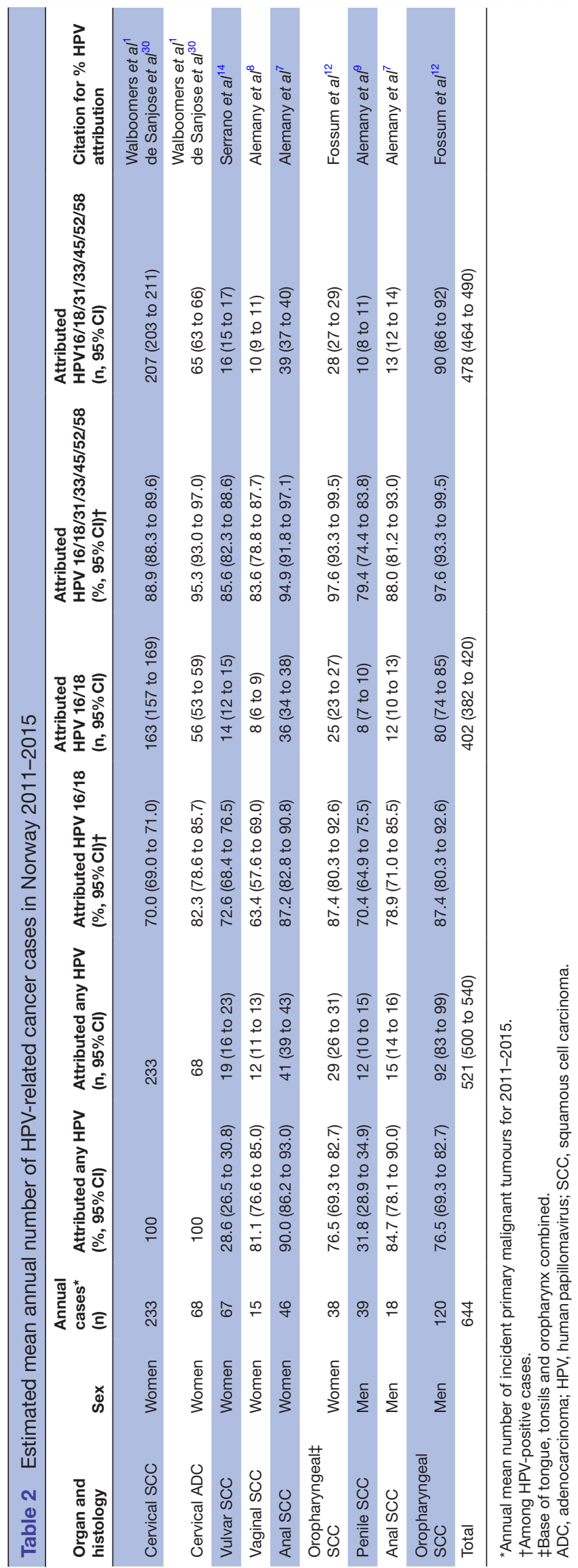


higher number of cancer cases compared with a vaccine that protects against HPV 16/18, most of which occur at the cervix.

Since approximately $50 \%$ of cervical precancer is associated with vaccine-type HPV, vaccination will also reduce the disease burden of precancer and the costs associated with cervical screening. ${ }^{44}$ In 2015 , close to 4000 women were treated for cervical precancer in Norway, and about 450000 screening smears were analysed. ${ }^{45}$ Moreover, HPV vaccines that protect against low-risk types 6 and 11 can prevent genital warts, which affect about $10 \%$ of the population, ${ }^{46}$ as well as the rare, but serious condition of respiratory papillomatosis.

We have assessed the potential for reducing HPV-related cancer through direct protection against the highrisk vaccine types included in the currently available HPV vaccines. However, the vaccines may also offer cross-protection against HPV types not included in the vaccines. A recent study on population-based HPV prevalence data found that the magnitude of cross-protection against HPV types 31, 33 and 45 was similar to protection against vaccine-type HPV 16 and 18 among women aged 20-21 who were vaccinated aged $12-13$ years. ${ }^{47}$ Thus, cross-protective effects may add to the protective potential estimated in the present study.

\section{Strengths and limitations}

The major strength of the present study is the accuracy and the scale of the cancer data presented.$^{26}$ Reporting of cancer is mandatory by law in Norway, and cancer registration is thus considered virtually complete. ${ }^{48}$ The underlying population data are also accurate and complete, which ensure that the incidence estimates are valid for the whole Norwegian population. Moreover, increased precision in addressing HPV-related cancers was achieved by excluding cancer cases with histological types that are not related to HPV. Another strength is the extensive study period for the assessment of temporal trends. However, this study also has several limitations. First, this is an observational study, and the registry data do not reveal what has caused the temporal changes in incidence documented here. Second, none of the HPV vaccines have yet proved effective against cancer. Because HPV-related cancer normally takes a decade or more to develop, the best evidence for vaccine effectiveness is still limited to preinvasive lesions and genital warts. Moreover, such evidence is strong for cervical lesions, but as yet, less data have accrued for non-cervical organs, and the currently available HPV vaccines are not yet approved for the prevention of cancer at all organs addressed in the present paper. However, knowledge about the natural history of HPV-related cancer progression makes subsequent vaccine-induced reductions in cancer likely, for any organ in which cancer is associated with vaccine-type HPV. Third, there is uncertainty regarding the type-specific HPV-attributable fraction for cancers in all organs, which reduces the accuracy of the estimates of the cancer cases preventable by HPV vaccination. This particularly applies to the non-cervical cancers, which are relatively rare and less studied, and which also may have a multifactorial aetiology and important risk factors additional to HPV. ${ }^{41} 4950$

\section{CONCLUSIONS}

In conclusion, the incidence of cervical SCC is limited by organised screening in Norway and has not changed significantly since 2004. In contrast, the other HPV-related cancers generally increase in incidence, and this change has been gradual and long-lasting. The increasing incidence trends, and the combined burden of the HPV-related cancers in Norway highlight the importance of HPV vaccination to prevent future cancer cases. HPV vaccination can prevent a substantial number of cancers, also at non-cervical sites and among men. The majority of the additional cancer cases that can be prevented by using a vaccine against HPV 16/18/31/33/45/52/58, compared with a vaccine against HPV $16 / 18$, occur in the cervix.

Acknowledgements We thank Bettina K Andreassen for statistical advice.

Contributors $\mathrm{BTH}, \mathrm{SC}$ and MN conceived and designed the study. SC managed and analysed the data. BTH drafted the manuscript. All authors critically reviewed manuscript drafts and approved the final version.

Funding This research received no specific grant from any funding agency in the public, commercial or not-for-profit sectors.

Competing interests The Cancer Registry of Norway has received grants from MSD Norway during the conduct of the study.

Patient consent Detail has been removed from this case description/these case descriptions to ensure anonymity. The editors and reviewers have seen the detailed information available and are satisfied that the information backs up the case the authors are making.

Provenance and peer review Not commissioned; externally peer reviewed.

Data sharing statement All data are available by request to the corresponding author.

Open Access This is an Open Access article distributed in accordance with the Creative Commons Attribution Non Commercial (CC BY-NC 4.0) license, which permits others to distribute, remix, adapt, build upon this work non-commercially, and license their derivative works on different terms, provided the original work is properly cited and the use is non-commercial. See: http://creativecommons.org/ licenses/by-nc/4.0/

(c) Article author(s) (or their employer(s) unless otherwise stated in the text of the article) 2018. All rights reserved. No commercial use is permitted unless otherwise expressly granted.

\section{REFERENCES}

1. Walboomers JM, Jacobs MV, Manos MM, et al. Human papillomavirus is a necessary cause of invasive cervical cancer worldwide. J Pathol 1999;189:12-19.

2. papillomaviruses IHIARC Monogr Eval Carcinog Risks Hum 2012;100B:255-313.

3. Parkin DM. The global health burden of infection-associated cancers in the year 2002. Int J Cancer 2006;118:3030-44.

4. Bernard HU, Burk RD, Chen Z, et al. Classification of papillomaviruses (PVs) based on 189 PV types and proposal of taxonomic amendments. Virology 2010;401:70-9.

5. Ma Y, Madupu R, Karaoz U, et al. Human papillomavirus community in healthy persons, defined by metagenomics analysis of human microbiome project shotgun sequencing data sets. J Virol 2014;88:4786-97.

6. Egawa N, Egawa K, Griffin H, et al. Human Papillomaviruses; Epithelial Tropisms, and the Development of Neoplasia. Viruses 2015;7:3863-90. 
7. Alemany L, Saunier M, Alvarado-Cabrero I, et al. Human papillomavirus DNA prevalence and type distribution in anal carcinomas worldwide. Int J Cancer 2015;136:98-107.

8. Alemany L, Saunier M, Tinoco L, et al. Large contribution of human papillomavirus in vaginal neoplastic lesions: a worldwide study in 597 samples. Eur J Cancer 2014;50:2846-54.

9. Alemany L, Cubilla A, Halec G, et al. Role of Human Papillomavirus in Penile Carcinomas Worldwide. Eur Urol 2016;69:953-61.

10. de Sanjosé S, Alemany L, Ordi J, et al. Worldwide human papillomavirus genotype attribution in over 2000 cases of intraepithelial and invasive lesions of the vulva. Eur $J$ Cancer 2013;49:3450-61.

11. Ndiaye C, Mena M, Alemany L, et al. HPV DNA, E6/E7 mRNA, and p16INK4a detection in head and neck cancers: a systematic review and meta-analysis. Lancet Oncol 2014;15:1319-31.

12. Fossum GH, Lie AK, Jebsen P, et al. Human papillomavirus in oropharyngeal squamous cell carcinoma in South-Eastern Norway: prevalence, genotype, and survival. Eur Arch Otorhinolaryngol 2017;274:4003-10.

13. Li N, Franceschi S, Howell-Jones R, et al. Human papillomavirus type distribution in 30,848 invasive cervical cancers worldwide: Variation by geographical region, histological type and year of publication. Int $J$ Cancer 2011:128:927-35.

14. Serrano B, de Sanjosé S, Tous S, et al. Human papillomavirus genotype attribution for HPVs $6,11,16,18,31,33,45,52$ and 58 in female anogenital lesions. Eur J Cancer 2015;51:1732-41.

15. Muñoz N, Manalastas R, Pitisuttithum P, et al. Safety, immunogenicity, and efficacy of quadrivalent human papillomavirus (types $6,11,16,18$ ) recombinant vaccine in women aged 24-45 years: a randomised, double-blind trial. Lancet 2009;373:1949-57.

16. Lehtinen M, Paavonen J, Wheeler CM, et al. Overall efficacy of HPV-16/18 AS04-adjuvanted vaccine against grade 3 or greater cervical intraepithelial neoplasia: 4-year end-of-study analysis of the randomised, double-blind PATRICIA trial. Lancet Oncol 2012;13:89-99.

17. Joura EA, Giuliano AR, Iversen OE, et al. A 9-valent HPV vaccine against infection and intraepithelial neoplasia in women. $N$ Engl $J$ Med 2015;372:711-23.

18. Garland SM, Kjaer SK, Muñoz N, et al. Impact and Effectiveness of the Quadrivalent Human Papillomavirus Vaccine: a systematic review of 10 years of real-world experience. Clin Infect Dis 2016:63:519-27.

19. Sasieni PD, Cuzick J, Lynch-Farmery E. Estimating the efficacy of screening by auditing smear histories of women with and without cervical cancer. The National Co-ordinating Network for Cervical Screening Working Group. Br J Cancer 1996;73:1001-5.

20. Lönnberg S, Hansen BT, Haldorsen T, et al. Cervical cancer prevented by screening: Long-term incidence trends by morphology in Norway. Int J Cancer 2015;137:1758-64.

21. Vaccarella S, Franceschi S, Engholm G, et al. 50 years of screening in the Nordic countries: quantifying the effects on cervical cancer incidence. Br J Cancer 2014;111:965-9.

22. Bray F, Carstensen B, Møller H, et al. Incidence trends of adenocarcinoma of the cervix in 13 European countries. Cancer Epidemiol Biomarkers Prev 2005;14:2191-9.

23. Parkin DM, Bray F. Chapter 2: The burden of HPV-related cancers. Vaccine 2006;24:S11-S25.

24. Baldur-Felskov B, Hannibal CG, Munk C, et al. Increased incidence of penile cancer and high-grade penile intraepithelial neoplasia in Denmark 1978-2008: a nationwide population-based study. Cancer Causes Control 2012;23:273-80.

25. Lai J, Elleray R, Nordin A, et al. Vulval cancer incidence, mortality and survival in England: age-related trends. BJOG 2014;121:728-38.

26. Larsen IK, Småstuen M, Johannesen TB, et al. Data quality at the Cancer Registry of Norway: an overview of comparability, completeness, validity and timeliness. Eur J Cancer 2009;45:1218-31.

27. Doll R, Payne P, Waterhouse J. Cancer incidence in five continents: a technical report. Berlin: Springer Verlag, 1966.
28. Kim HJ, Fay MP, Feuer EJ, et al. Permutation tests for joinpoint regression with applications to cancer rates. Stat Med 2000;19:335-51.

29. Clegg LX, Hankey BF, Tiwari R, et al. Estimating average annual per cent change in trend analysis. Stat Med 2009;28:3670-82.

30. de Sanjose S, Quint WG, Alemany L, et al. Human papillomavirus genotype attribution in invasive cervical cancer: a retrospective cross-sectional worldwide study. Lancet Oncol 2010;11:1048-56.

31. Cancer in Norway 2015. Oslo: Cancer Registry of Norway 2016.

32. Islami F, Ferlay J, Lortet-Tieulent J, et al. International trends in anal cancer incidence rates. Int J Epidemiol 2017;46:dyw276.

33. Chaturvedi AK, Anderson WF, Lortet-Tieulent J, et al. Worldwide trends in incidence rates for oral cavity and oropharyngeal cancers. $J$ Clin Oncol 2013;31:4550-9.

34. Arya M, Li R, Pegler K, et al. Long-term trends in incidence, survival and mortality of primary penile cancer in England. Cancer Causes Control 2013;24:2169-76.

35. Stigum H, Samuelsen SO, Traeen B. Analysis of first coitus. Arch Sex Behav 2010;39:907-14.

36. Johnson AM, Mercer CH, Erens B, et al. Sexual behaviour in Britain: partnerships, practices, and HIV risk behaviours. Lancet 2001;358:1835-42.

37. Bajos N, Bozon M, Beltzer N, et al. Changes in sexual behaviours: from secular trends to public health policies. AIDS 2010;24:1185-91.

38. Liu G, Hariri S, Bradley H, et al. Trends and patterns of sexual behaviors among adolescents and adults aged 14 to 59 years, United States. Sex Transm Dis 2015;42:20-6.

39. Hansen BT, Kjaer SK, Arnheim-Dahlstrom L, et al. Trends in age at first intercourse and number of sexual partners among women in Denmark, Norway and Sweden. 31st International Papillomavirus Conference. Cape Town, 2017.

40. Daling JR, Madeleine MM, Johnson LG, et al. Human papillomavirus, smoking, and sexual practices in the etiology of anal cancer. Cancer 2004;101:270-80.

41. D'Souza G, Kreimer AR, Viscidi R, et al. Case-control study of human papillomavirus and oropharyngeal cancer. $N$ Engl $J$ Med 2007;356:1944-56.

42. International Collaboration of Epidemiological Studies of Cervical Cancer. Cervical carcinoma and sexual behavior: collaborative reanalysis of individual data on 15,461 women with cervical carcinoma and 29,164 women without cervical carcinoma from 21 epidemiological studies. Cancer Epidemiol Biomarkers Prev 2009;18:1060-9.

43. Vink MA, Bogaards JA, van Kemenade FJ, et al. Clinical progression of high-grade cervical intraepithelial neoplasia: estimating the time to preclinical cervical cancer from doubly censored national registry data. Am J Epidemiol 2013;178:1161-9.

44. Kim JJ, Burger EA, Sy S, et al. Optimal Cervical Cancer Screening in Women Vaccinated Against Human Papillomavirus. J Natl Cancer Inst 2017;109:1-9.

45. Årsrapport K. Masseundrsøkelsen mot livmorhalskreft (Yearly report of the Norwegian cervical cancer screening program). Oslo, 2015.

46. Kjaer SK, Tran TN, Sparen P, et al. The burden of genital warts: a study of nearly 70,000 women from the general female population in the 4 Nordic countries. J Infect Dis 2007;196:1447-54

47. Kavanagh K, Pollock KG, Cuschieri K, et al. Changes in the prevalence of human papillomavirus following a national bivalent human papillomavirus vaccination programme in Scotland: a 7-year cross-sectional study. Lancet Infect Dis 2017;17:1293-302.

48. Bilet EF, Langseth $\mathrm{H}$, Thoresen $\mathrm{S} \varnothing$, et al. Completeness of invasive cervical cancer at the Cancer Registry of Norway. Acta Oncol 2009;48:1070-3.

49. Miralles-Guri C, Bruni L, Cubilla AL, et al. Human papillomavirus prevalence and type distribution in penile carcinoma. J Clin Pathol 2009;62:870-8

50. van der Avoort IA, Shirango H, Hoevenaars BM, et al. Vulvar squamous cell carcinoma is a multifactorial disease following two separate and independent pathways. Int J Gynecol Pathol 2006;25:22-9. 